\title{
An Easy and Practical Method for Routine, Bedside Testing of Somatosensory Systems in Extremely Low Birth Weight Infants
}

\author{
SAMPSA VANHATALO, VEIKKO JOUSMÄKI, STURE ANDERSSON, AND MARJO METSÄRANTA
}

Department of Clinical Neurophysiology [S.V.] and Department of Pediatrics [S.A., M.M.], Helsinki University Central Hospital, Helsinki, Finland; Brain Research Unit [V.J.], Helsinki University of Technology, Espoo, Finland

\begin{abstract}
This study was set out to develop and describe a novel, simple, and safe method for routine bedside testing of somatosensory system in very early preterm infants. We recorded electroencephalogram (EEG) activity after tactile stimulation of hand (palm) and foot (sole) by a soft hairbrush stimulator in extremely low birth weight infants $(n=10 ; \mathrm{GA}, 24-28$, recording at conceptional age 30-32 wk) and compared with the raw EEG responses to those seen by one- or two-channel brain monitors. In every subject, single tactile stimuli produced prominent $(100-350 \mu \mathrm{V})$ somatosensory evoked responses (SERs) that were readily identified in the ongoing EEG signal. The maximal SER was in the contralateral hemisphere at around the corresponding somatosensory representation areas. Conventional EEG filtering did significantly reduce the SERs, but they could still be identified in the routine brain monitor setting widely available in NICUs. The method described here is directly applicable to assessment of integrity of somatosensory system in the early preterm period. It needs minimal training and requires an EEG system or a brain monitor device that is available in most units. Thus, the technique is likely to open a novel window to neurologic assessment of these babies. (Pediatr Res 66: 710-713, 2009)
\end{abstract}

$\mathrm{D}$ espite major advances in cardiorespiratory care and subsequent improvement in survival rates through the stay in the NICU, a major proportion of the extremely low birth weight (ELBW) infants develop with debilitating neurocognitive dysfunctions $(1,2)$. The etiological mechanisms in some problems may be obvious, such as major brain lesions caused by intracranial hemorrhages, which are routinely captured by brain ultrasound examination. Because of the immaturity of nervous system, especially of the corticospinal connectivity, clinical examinations cannot detect whether a possible brain lesion is associated with damage to the thalamocortical connections responsible for conveying (motor and) sensory information. Studies on preterm infants at full-term age have shown that assessment of the integrity of these pathways by somatosensory evoked potential (SEP) provides valuable prognostic information $(3,4)$. For an early diagnosis, and even for a potential early intervention, it would be of high interest to have a possibility to assess somatosensory pathways immediately after vascular lesions, i.e. during the early postnatal weeks at around 28-32 of conceptional age.

Received April 24, 2009; accepted August 25, 2009.

Correspondence: Sampsa Vanhatalo, M.D., Ph.D., Department of Clinical Neurophysiology, Helsinki University Central Hospital (HUCH), P.O. Box 280, FIN-00029 HUS, Finland; e-mail: sampsa.vanhatalo@helsinki.fi

Supported by the University Hospital of Helsinki, the Finnish Academy, and the Sigrid Jusélius Foundation.
In this context, it is unfortunate that the conventional SEP responses are obtained in only a variable proportion of preterm babies $(5,6)$, discouraging their use as a routine monitoring tool. Several studies have provided explanations for the failure of conventional SEP paradigms in preterm infants. In essence, the conventional SEP paradigms are just modifications from adult SEP recordings, which assume that cortical responses are rapid, constant in shape, and they do not show a notable fatigue to fast repeating stimuli. Because of the already known variability, time scales, and other characteristics of the sensory systems during early development, the current SEP parameters (stimulation frequency, filters, time windows, and averaging) are likely to effectively hamper if not preclude observation of the brain responses characteristic of human preterm brain (7).

In this article, we describe a method for eliciting cortical reactions to somatosensory stimuli in a way that complies with all the known physiology and anatomy of preterm brain. The method is able to yield reliable and reproducible cortical reactions in ELBW infants as early as 28 wk of conceptional age. Most importantly, it was designed to be applicable as such for bedside studies at settings where only clinicians have an access to basic electroencephalogram (EEG) recording device or a long-term brain monitor with a "raw EEG" display such as a digital amplitude-integrated EEG device [aEEG; also called cerebral function monitor (CFM), $(8,9)]$.

\section{MATERIALS AND METHODS}

Subjects. Ten infants (conceptional age at recording, 30-32 wk; GA, 24-28 wk; Table 1) were included in this study from a larger ongoing research project that develops EEG methodology and examines the CNS development in this age group. None of these babies had ultrasound abnormalities. Informed consent was obtained from the parents, and the study was approved by the Ethics Committee of the Hospital for Children and Adolescents, Helsinki University Central Hospital.

EEG-recording and stimulator device. EEG signal was recorded at 256 $\mathrm{Hz}$ with a video-synchronized, 32-channel EEG device (NicOne, Magnus amplifier; Cardinal Healthcare, USA), which has a time constant of $10 \mathrm{~s}$ and hence enables relatively reliable recordings of slow events up to few seconds [nominal hardware cutoff at $0.016 \mathrm{~Hz}(10)$ ]. Electrodes [sintered $\mathrm{Ag} / \mathrm{AgCl}$; (11)] were placed according to the international 10-20 standard, attached either individually or in an electrode cap (Waveguard; ANT-Neuro, Germany; www.ant-neuro.com).

Stimulator device was modified from an adult tactile stimulator recently introduced by Jousmäki et al. (12). The handle of this baby device was taken

\footnotetext{
Abbreviations: aEEG, amplitude-integrated EEG; CFM, cerebral function monitor; SEP, somatosensory evoked potential; SER, somatosensory evoked response
} 
Table 1. Clinical information of the children included in the study

\begin{tabular}{|c|c|c|c|c|c|c|}
\hline $\mathrm{nr}$ & Gestational age $(w k+d)$ & Birth weight $(\mathrm{g})$ & Sex & Conceptional age at study & Postnatal age at study (d) & Other diagnoses \\
\hline 1 & $23+6$ & 520 & $\mathrm{~F}$ & $30+4$ & 40 & ROP, BPD \\
\hline 2 & $26+0$ & 780 & M & $30+1$ & 29 & $\mathrm{BPD}$, twin \\
\hline 3 & $26+0$ & 780 & M & $32+0$ & 42 & ROP, BPD, twin \\
\hline 4 & $26+0$ & 590 & $\mathrm{~F}$ & $30+4$ & 32 & BPD \\
\hline 5 & $26+5$ & 930 & M & $30+3$ & 26 & \\
\hline 6 & $26+5$ & 940 & M & $32+3$ & 40 & \\
\hline 8 & $26+3$ & 640 & $\mathrm{~F}$ & $31+5$ & 37 & BPD \\
\hline 9 & $27+6$ & 860 & M & $31+0$ & 22 & \\
\hline 10 & $26+4$ & 1120 & M & $30+1$ & 24 & BPD \\
\hline 11 & $26+4$ & 1120 & M & $30+5$ & 29 & \\
\hline
\end{tabular}

BDP, bronchopulmonary dysplasia; ROP, retinopathy; M, male; F, female.

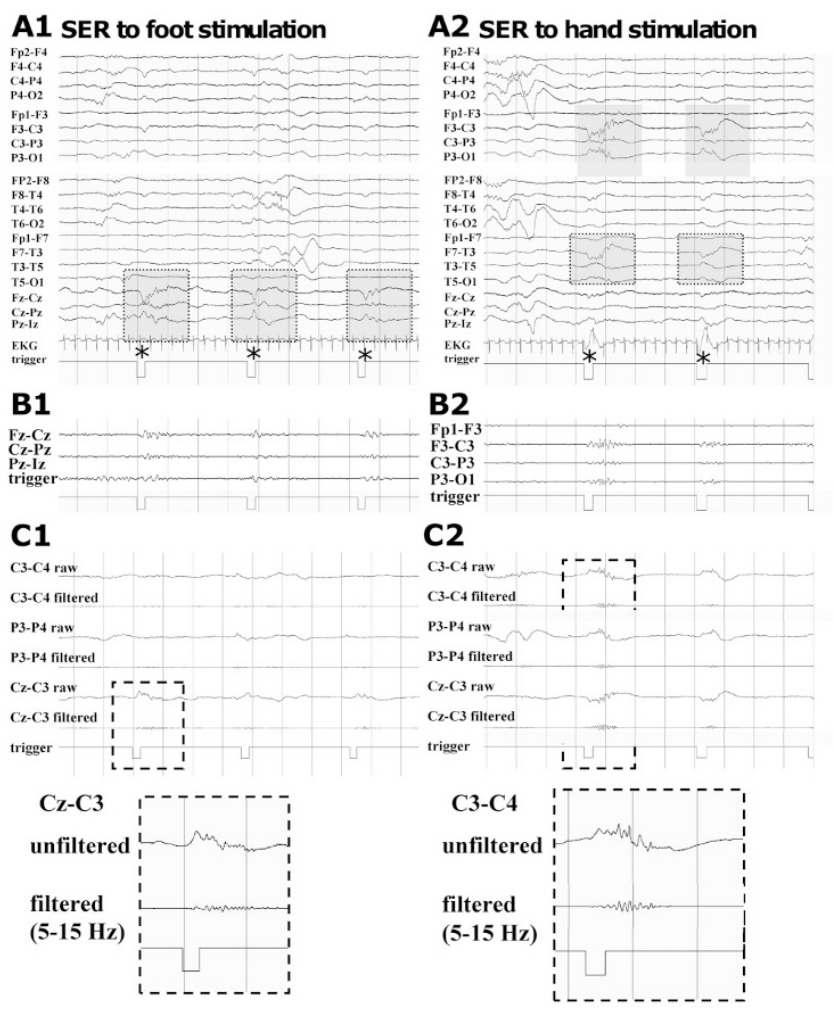

Figure 1. The unfiltered and conventionally filtered SER to foot and hand stimuli. Each trace shows individual SERs. This figure summarizes all findings from both foot (left column, A1-C1) and hand (right column, A2-C2) stimulations, respectively. Square signal in the trigger channel (marked with asterisks) depict the timing of stimulation. In the upper plates ( $A 1$ and $A 2)$, all channels of the whole EEG recording is shown without filtering, with gray boxes drawn over the visually identified SER responses. In the middle (B1 and $B 2$ ), selected traces are shown with a conventionally filtered SERs (lowcut/ highpass $0.5 \mathrm{~Hz}$ ). In the bottom traces ( $C 1$ and $C 2$ ), the channels are remontaged to electrode pairs that are seen in a typical aEEG/CFM monitor, and these traces are shown both as a raw (unfiltered) and as filtered $(5-15 \mathrm{~Hz}$, which roughly mimics aEEG bandpass). Finally, a magnification of a single response is shown in the inset taken from the Figures $C 1$ and $C 2$.

from a cosmetic brush, and there is an optical fiber bundle that goes through the brush with its tip adjusted to the level of brush hairs. Touch of the brush onto baby skin is detected by a modulated red light $(680 \mathrm{~nm})$ that is emitted by half of the fibers, whereas the reflection from the skin is detected by the other half of the fibers (emission and detection by a photoelectric switch that runs with batteries, Omron E3×-N41, Japan). When a defined reflection threshold is exceeded, the device will send a trigger signal to the EEG amplifier to be recorded as a square pulse in one channel (Fig. 1). In addition, we also performed standard, electrical stimulation of the right median nerve in one baby (conceptional age $31.7 \mathrm{wk}$; stimulation intensity high enough to trigger a thumb twitch). These responses were compared with the somato- sensory evoked responses (SERs) elicited by tactile stimuli (see later), and to the conventional SEPs reported in the literature.

Performance of the stimulation. Stimulation was performed manually by a gentle but determined brush on the palm or the sole (see an online video at http://www.helsinki.fi/science/eeg/tSEP_video/tSEP demo.wmv). Occasionally, we also tried touching with bare examiner's finger which yielded comparable responses. We found it to be important that stimulus was given at a time when not much other EEG activity was present $(13,14)$, and when no other limb movements were seen in the baby. It is obvious that both active (caused by the baby) and passive (caused by the clinician) body movements result in a sensory feedback from tendons and skin receptors (14-16). Thus, the "specificity" of the EEG response to the given tactile stimulus is guaranteed only when the stimulated limb does not move more than the gentle push by the brush. To avoid movement, we often used gentle limb fixation by pressing the sleeve against the mattress. A minimum of three successful responses were analyzed from each limb. We defined the response as being absent if more than five stimuli were delivered $>3 \mathrm{~s}$ after the latest spontaneous EEG burst, but not a visually detectable EEG responses were seen. With these criteria, all babies showed responses, but because of the complex relationship between spontaneous and evoked activities (13), we chose not to quantify this in more detail.

Assessment of the somatosensory responses. All EEG signals were assessed by visual analysis. Unfiltered EEG signals were then compared with signals that mimic recordings with the typical one- or two-channel aEEG/ CFM device, i.e., we made a new montage offline. In addition, we used digital high-pass filters offline that cut out the slowest components and hence mimic the typical filter settings $[0.5$ or $1.0 \mathrm{~Hz},(17)$ and $5-15 \mathrm{~Hz}(8,9)]$, in the conventional neonatal or in the aEEG/CFM recordings. Please note that the resulting "passband" from this filter combination may not be completely identical to aEEG, which also varies between commercial aEEG devices. However, these filter values are obtained from the standard filter repertoire of our EEG device, and it results in very similar visual appearance of the traces.

\section{RESULTS}

We found a robust, high amplitude, and long duration SER to tactile stimulation in every baby examined. The location of the response was always over central cortical areas that closely correspond to the primary somatosensory area. Hand stimulation resulted in largest responses in the central leads (C3 and C4; followed by the temporal T3/T4 leads), with a somewhat variable onset $[95 \pm 7.3 \mathrm{~ms}(\mathrm{SEM})]$, duration of about $1-2 \mathrm{~s}(1.5 \pm 0.45 \mathrm{~s}$; data pooled from both sides from 10 babies; reference $\mathrm{Fz}$ ) and an amplitude ranging between $100-350 \mu \mathrm{V}(232 \pm 57 \mu \mathrm{V})$. Foot stimulation resulted in a less prominent but still very reliable response in the central leads $(\mathrm{Pz}$ and $\mathrm{Cz})$, with a somewhat variable onset $(107 \pm 3.8$ $\mathrm{ms})$, with a length of about $1-2 \mathrm{~s}(1.54 \pm 0.32 \mathrm{~s})$, and an amplitude up to $150 \mu \mathrm{V}(128 \pm 63 \mu \mathrm{V})$. The response waveforms for both hand and foot stimuli were always little variable (Fig. 2). They were also smaller $(p<0.01)$ and later $(p<0.05)$, but not shorter $(p=0.81)$ to foot stimuli compared with hand stimuli. However, the overall pattern was invariably 


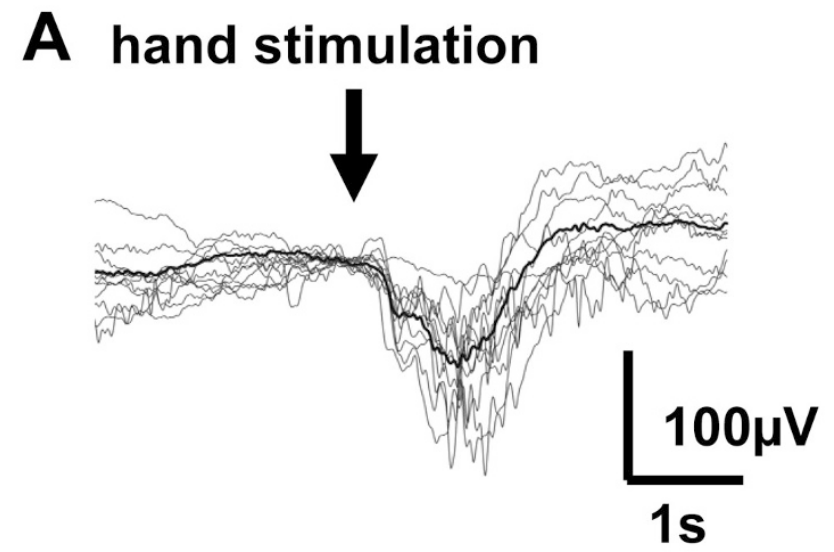

Figure 2. Trial-to-trial variation of the SER response. Ten individual, consecutive responses after hand stimulation (derivation $\mathrm{C} 4-\mathrm{Fz}$ ) are shown together with their average.

present, consisting of a slow "carrier wave" with a superimposed faster activity riding on it.

Although SERs were seen in every baby tested, there were individual stimuli (trials), which did not result in a visible EEG response. This was the case especially if the stimulus was given too shortly after a preceding endogenous (spontaneous) cortical EEG event (see also Refs. 13 and 14) or if the baby was moving his/her limbs. Namely, we observed that an active body movement did also elicit EEG events that closely resembled those SERs seen after deliberate stimulation of hand and foot (14).

Regarding a practical possibility to see SERs in a routine brain monitoring, it was intriguing that these responses were clearly seen at a single-trial level, and that they could be identified online by watching the raw EEG signal. Next, we examined whether it was possible to detect these responses by the typical aEEG/CFM devices used worldwide in NICUs $(8,9)$. These devices are limited in the number of channels available (one or two), as well as in their frequency response designed to filter out the slowest signal components (10), i.e. the hallmarks of SERs we describe here $(15,16)$.

In Figure 1, we show that the Fz-C3 (or Fz-C4; for the right and left limb stimulation, respectively) montages typically used for aEEG/CFM recordings are able to show the SERs to hand stimulation, whereas a midline electrode is needed to show the SERs to foot stimulation. A single-channel derivation between $\mathrm{C} 3-\mathrm{C} 4$ is obviously able to show responses to both sides, but it is unable to distinguish reliably between left and right side responses.

We show further how the SERs are strongly attenuated by filter settings typically used for aEEG/CFM monitoring (Fig. 1). The salient slow component is practically lost after filtering with the typical aEEG/CFM bandwidth (around 2-15 Hz). The response can be still fairly well identified from traces where the high-pass filter has been set to conventional EEG settings at $0.3-1.0 \mathrm{~Hz}$ (17). Hence, although visual identification of the SERs from a raw EEG is easy even without knowing the exact timing of stimulation, the stimulation time marks (triggers) must be precise to be able to analyze SERs from a trace that has been distorted by filters. Distortion of signals by such

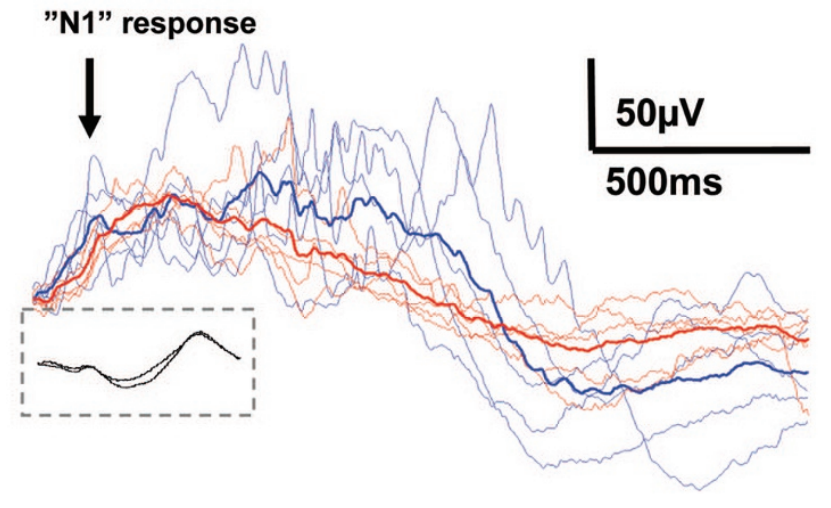

Figure 3. Comparison between SER responses elicited by tactile (blue) and electric (red) stimulation as well as a conventional SEP response (square box), reprinted from Tombini $M$ et al. Clin Neurophyseal 120:783-789, copyright (C) 2008 International Federation of Clinical Neurophysiology, with permission. Five individual responses (thinner lines) and their corresponding average (thick line) show that, there is a high variability in individual responses, but still a little N1 response can be seen in the average wave form, which is comparable with the N1 in conventional SEPs (square box). All traces are fitted to the same time-amplitude scale, which makes it clear that SERs are many times bigger and larger than conventional SEPs. The little different "tilting" of the conventional SEP response is due to the highpass filter (at $1 \mathrm{~Hz}$ ) used in conventional SEP recordings as opposed to no digital filtering used in our study.

filters does indeed preclude analysis of the most prominent component, the slow "carrier wave," which may carry important information about the underlying cortical and subcortical structures (such as subplate function, 7, 13, 18).

Finally, we wanted to compare tactile SERs with responses evoked by electrical stimuli in the single-trial manner (as tactile SERs described here) or those obtained by the conventional SEP paradigm. We were able to deliver individual electric stimuli to one baby, and we found that electrical stimuli result in qualitatively comparable individual SERs (Fig. 3). Their overall shape tended to be little flatter than those seen after tactile stimuli. We did not have particular permission to record conventional SEPs from these babies, but comparison of SERs and conventional SEPs, as taken from the recent literature, showed that $\mathrm{N} 1$ response can be seen in all forms of somatosensory responses (see Fig. 3).

\section{DISCUSSION}

In this study, we describe a novel method that is practical and easy enough to be used as a routine tool in NICUs to examine function of central somatosensory system during early conceptional weeks. Our observations are fully in line with the previous studies (14-16) that have demonstrated how EEG activity shows a visually prominent combination of slow and "burst-kind" faster components after sensory stimulus to peripheral body parts. Our main addition to the existing knowledge is that we introduce a method applicable for bedside testing by any clinician (i.e. no specialist training is needed), and that we assess the plausibility of studying SERs by using routine brain monitors instead of conventional EEG systems. 
In addition to the technical practicality, we also demonstrate here that our somatosensory testing paradigm makes it possible to record safely, so that the physiologic stress to even the youngest babies is reduced to level of basic care. We feel that this may be of major importance in preterm EEG practice, because a major bottleneck in providing fluent EEG service to preterm units has been the lack of practical and patientfriendly stimulation techniques. These together imply that testing of somatosensory function may readily become a routine part of clinical examination in babies that are undergoing long-term EEG monitoring.

From the comparison of SERs to conventional SEPs (Fig. 3), it becomes clear that the N1 component typically measured in preterm SEPs is present in both responses. This comparison does, however, reveal two very relevant aspects of preterm somatosensory physiology: First, the main component of cortical somatosensory reaction is far beyond the conventional time and amplitude window, and it is highly variable in form, hence the term "response" as opposed to "potential." Second, even the first cortical component, N1, is variable and not at all visible in all cases, which by default results in diminishing if not elimination of the averaged waveform. Indeed, this may readily explain the reason why recording of conventional SEPs from early preterm babies tends to fail in so high proportions of cases (18).

The waveform components of SERs have been studied in several in vivo and in vitro animal models (7). Current literature suggests that the slow component requires a concerted action of the subplate and cortex, and its presence reflects the maturational stage where thalamocortical fibers are just about to establish their afferent cortical connections. By offering a putative functional test for subplate, SERs are opening a completely new window to a very important $(19,20)$ but as yet unapproachable structure in live preterm human brain.

It is intriguing that a proper, spontaneous brain activity is necessary for both the structural and functional brain development (20-23). A wide range of animal studies have shown that early brain activity is exquisitely sensitive to a large number of challenges, many of which may also be encountered during neonatal intensive care treatment $(19,20,24)$. Hence, it would be most rational to include assessment of this kind into the routine procedure in preterm neurologic evaluation, especially as a part of routine brain monitoring.

Finally, our study demonstrates that recording of even a "high fidelity" (HiFi) EEG [i.e. high density caps, Full-band (FbEEG), and evoked activity (25)] is practical in the neonatal incubator environment. These technically trivial modifications in the recording and analysis techniques make it possible to significantly increase the yield of neonatal EEG studies. Most importantly, recordings of this kind enable interpretation of the preterm EEG in a physiologic context $(13,24)$, and open a window to functional assessment of the as yet elusive subcortical (i.e., subplate) mechanisms $(19,20)$. A thorough assessment of the clinical utility of SERs described here will need wider scale, systematic studies including sick babies and their outcome measures. Such studies can only be accomplished by having a methodology that is available to every bedside clinician at all times during NICU care.
Acknowledgments. We thank the parents who gave permissions to conduct the study on their babies as well as the neonatal nurses and neurophysiology technicians who were helpful in setting up the recordings.

\section{REFERENCES}

1. Marlow N, Wolke D, Bracewell MA, Samara M; EPICure Study Group 2005 Neurologic and developmental disability at six years after extremely preterm birth. N Engl J Med 352:9-19

2. Tommiska V, Heinonen $K$, Kero $P$, Pokela ML, Tammela $O$, Järvenpää AL, Salokorpi T, Virtanen M, Fellman V 2003 A national two year follow up study of extremely low birthweight infants born in 1996-1997. Arch Dis Child Fetal Neonatal Ed 88:F29-F34

3. Pike AA, Marlow N 2000 The role of cortical evoked responses in predicting neuromotor outcome in very preterm infants. Early Hum Dev 57:123-135

4. de Vries LS, Eken P, Pierrat V, Daniels H, Casaer P 1992 Prediction of neurodevelopmental outcome in the preterm infant: short latency cortical somatosensory evoked potentials compared with cranial ultrasound. Arch Dis Child 67:1177-1181

5. Taylor MJ, Boor R, Ekert PG 1996 Preterm maturation of the somatosensory evoked potential. Electroencephalogr Clin Neurophysiol 100:448-452

6. Smit BJ, Ongerboer de Visser BW, de Vries LS, Dekker FW, Kok JH 2000 Somatosensory evoked potentials in very preterm infants. Clin Neurophysiol 111:901-908

7. Vanhatalo S, Lauronen L 2006 Neonatal SEP — back to bedside with basic science. Semin Fetal Neonatal Med 11:464-470

8. Hellström-Westas L, Rosen I, de Vries LS, Greisen G 2006 Amplitude integrated EEG classification and interpretation in preterm and term infants. NeoReviews 7:e76-e87

9. Hellström-Westas L, Rosén I 2006 Continuous brain-function monitoring: state of the art in clinical practice. Semin Fetal Neonatal Med 11:503-511

10. Vanhatalo S, Voipio J, Kaila K 2005 Full-band EEG (FbEEG): an emerging standard in electroencephalography. Clin Neurophysiol 116:1-8

11. Tallgren P, Vanhatalo S, Kaila K, Voipio J 2005 Evaluation of commercially available electrodes and gels for recording of slow EEG potentials. Clin Neurophysiol 116:799-806

12. Jousmäki V, Nishitani N, Hari R 2007 A brush stimulator for functional brain imaging. Clin Neurophysiol 118:2620-2624

13. Vanhatalo S, Kaila K 2009 Spontaneous and evoked activity in the early human brain. In: Lagercrantz H, Hanson MA, Ment LR, Peebles DM (eds) The Newborn Brain: Neuroscience and Clinical Applications. 2nd ed. Cambridge University Press, in press

14. Milh M, Kaminska S, Huon C, Lapillonne A, Ben-Ari Y, Khazipov R 2007 Rapid cortical oscillations and early motor activity in premature human neonate. Cereb Cortex 17:1582-1594

15. Hrbek A, Karlberg P, Olsson T 1973 Development of visual and somatosensory evoked responses in pre-term newborn infants. Electroencephalogr Clin Neurophysiol 34:225-232

16. Hrbek A, Hrbkova M, Lenard HG 1969 Somato-sensory, auditory and visual evoked responses in newborn infants during sleep and wakefulness. Electroencephalogr Clin Neurophysiol 26:597-603

17. Lamblin MD, Andre M, Challamel MJ, Curzi-Dascalova L, d'Allest AM, De Giovanni E, Moussalli-Salefranque F, Navelet Y, Plouin P, Radvanyi-Bouvet MF, Samson-Dollfus D, Vecchierini-Blineau MF 1999 [Electroencephalography of the premature and term newborn. Maturational aspects and glossary]. Neurophysiol Clin 29:123-219 [in French]

18. Tombini M, Pasqualetti P, Rizzo C, Zappasodi F, Dinatale A, Seminara M, Ercolani M, Rossini PM, Agostino R 2009 Extrauterine maturation of somatosensory pathways in preterm infants: a somatosensory evoked potential study. Clin Neurophysiol 120:783-789

19. Kostović I, Judas M 2007 Transient patterns of cortical lamination during prenatal life: do they have implications for treatment? Neurosci Biobehav Rev 31:1157-1168

20. Kanold PO 2009 Subplate neurons: Crucial regulators of cortical development and plasticity. Front Neuroanat 3:16. Epub 2009 Aug 20

21. Pallas SL 2001 Intrinsic and extrinsic factors that shape neocortical specification. Trends Neurosci 24:417-423

22. Penn AA, Shatz CJ 1999 Brain waves and brain wiring: the role of endogenous and sensory-driven neural activity in development. Pediatr Res 45:447-458

23. Price DJ, Kennedy H, Dehay C, Zhou L, Mercier M, Jossin Y, Goffinet AM, Tissir F, Blakey D, Molnár Z 2006 The development of cortical connections. Eur J Neurosci 23:910-920

24. Vanhatalo S, Kaila K 2006 Ontogenesis of EEG activity: from phenomenology to physiology. Semin Fetal Neonatal Med 11:471-478

25. Vanhatalo S, Metsäranta M, Andersson S 2008 High-fidelity recording of brain activity in the extremely preterm babies: Feasibility study in the incubator. Clin Neurophysiol 119:439-445

26. Tombini M, Pasqualetti P, Rizzo C, Zappasodi F, Dinatale A, Seminara M, Ercolani M, Rossini PM, Agostino R 2009 Extrauterine maturation of somatosensory pathways in preterm infants: A somatosensory evoked potential study. Clin Neurophysiol 120:783-789 